
\title{
25 Research Sourere \\ Effects of Prosthetic Design Parameters on Running of Unilateral Transfemoral Amputee
}

Cem Guzelbulut ( $\nabla$ cem@struct.t.u-tokyo.ac.jp )

The University of Tokyo https://orcid.org/0000-0001-9618-4032

\section{Katsuyuki Suzuki}

The University of Tokyo

\section{Satoshi Shimono}

The KAITEKI Institute, Inc.

\section{Hiroaki Hobara}

National Institute of Advanced Industrial Science and Technology

\section{Research Article}

Keywords: Amputee running, running-specific prosthesis, prosthetic parameters, running performance

Posted Date: May 21st, 2020

DOI: https://doi.org/10.21203/rs.3.rs-29522/v1

License: (c) (1) This work is licensed under a Creative Commons Attribution 4.0 International License. Read Full License 


\section{Abstract}

Carbon fiber running-specific prostheses (RSP) are widely used among lower-limb amputee runners. However, which prosthesis provides the best performance for a runner remains as an unanswered question. In this purpose, a computational model of the human body with prosthesis was created and the effect of prosthetic parameters on performance was investigated. Firstly, motion capture systems were used to collect data from the amputee running motion. Marker data and force plate data were obtained to create a digital human model. Kinematic data such as length of limbs, joint angles, etc. were calculated by using marker data. Then, inertial properties were estimated to conduct forward and inverse dynamic analyses. After building a computational model of amputee sprinting, joint positions and ground reaction forces (GRF) were compared with experimental results. The design parameters of the prosthesis were introduced to understand the effect of prosthesis on motion and performance. Response surface method was used to express motion adaption regarding geometry and stiffness of the prosthesis. Hip and knee sagittal joint angles were updated based on the response surface method to simulate joint motion adaptations of prosthesis worn. Then, average horizontal velocity, horizontal velocity change over one period, vertical and horizontal impulse was considered as performance functions. An evaluation parameter was proposed to generalize the idea of performance. Prosthetic knee moment and closest point of the prosthesis to the ground during the swing phase were defined as design constraints to consider knee-buckling and tripping of the prosthetic leg, respectively. The effect of design parameters on the performance and constraint functions was investigated. A method to determine and design suitable prostheses for an individual was proposed. It was revealed that the selection and design of prostheses holds an important place to increase performance.

\section{Introduction}

Running-specific prosthesis (RSP) have been developed during the last two decades and it is revealed that shape/stiffness regulation of the RSP may provide better sprint performances for individuals with lower extremity amputation [1]. Previous studies utilized mass-spring models [2], multibody models [3], and finite element models [4] to investigate the effect of prosthesis on the running mechanics, where contact time [5], leg stiffness [5-6], mechanical energy flows [7] were evaluated. Although these studies identified how the running mechanics was varied by wearing RSP, little is known about how the RSP should be designed for individuals with lower extremity amputation.

To understand the effect of RSP design (e.g. shape and stiffness) on the biomechanics and sprint performance, a computational multibody model of the runner with RSP was developed together with biomechanical data. For example, previous studies utilized ground reaction forces (GRFs) from forceinstrumented treadmill [8] and joint kinematics/kinetics from 3-D motion capture systems [9]. However, computational methods with experimental data often lead complicated design strategies. For example, it is hard to determine the location of the representative RSP ankle joint to carry out inverse dynamic analyses [10]. Therefore, in the present study, the RSP was considered as a multi-link structure consisting of rigid links with rotational springs and dampers to include deformation characteristics of the RSP. 
Further, since GRFs could also be affected by the mechanical properties of RSP, digital human models to estimate GRF [11] and contact models [12] have been used in forward and inverse dynamic analyses, respectively.

Another methodological issue to consider RSP design is the definitive evaluation of the sprint performance. Although metabolic cost, joint loading [13], gait asymmetry [14] were commonly used parameters in past studies, we used GRF impulses and horizontal velocity as performance indexes to figure out the effect of RSP on sprint performance. Further, we also considered to reduce the risk of 'knee buckling (defined as the sudden loss of postural support across the knee at a time of weight bearing)' induced by amount of internal knee flexion moments [15] and tripping at mid-swing phase for RSP design.

The purpose of the study was to establish a design methodology based on prosthetic parameters by considering the effect of the design on the motion of joints and the performance of runner. A computational human model has been built, and forward/inverse dynamic analyses have been carried out. Horizontal velocity and GRFs were calculated through the computational human model with different RSP and generated motions based on prostheses that were worn by the runner. The effect of each design parameter on performance and constraints were discussed. In this study, a comprehensive parametric design method for the RSP is proposed to improve the performance in individuals with lower extremity amputation.

\section{Methods And Modelling Subject Characteristics}

A female unilateral transfemoral amputee (Age 21 years old, Height $1.56 \mathrm{~m}$, Mass $58.3 \mathrm{~kg}$ ) participated in this study. The participant was informed about the experiment and the purpose of research and informed consent was obtained. Ethical Committee of the University of Tokyo/Office for Life Science Research Ethics and Safety approved the research. The participant performed trials by using the same prosthetic knee joint (3S80, Ottobock, Duderstadt, Germany) and different RSP blades.

\section{Experimental Procedures and Data Collection}

After adequate warm-up exercises, the participant carried out maximum sprinting on an indoor 40-meter straight path. To collect 3-D coordinate positions of the body and GRFs, motion capture system (20 cameras, VICON MX, sampled at $200 \mathrm{~Hz}$ ) and seven force plates (AMTI, sampled at $1000 \mathrm{~Hz}$ ) were positioned around 22-meter from starting line. Helen-Hayes marker set [16] was adopted to place markers on the anatomical part of the body as well as prosthetic knee and RSP. Trials in which the participant stepped on within the boundaries of force plates were accepted as successful trials. Four successful trials were obtained for each prosthetic blade. Enough rest time was provided between each trial. 


\section{Computational Model of Human with Running-Specific Prosthesis}

The raw GRFs and three-dimensional marker positions were filtered using a fourth-order zero-lag low-pass Butterworth filter with cut-off frequencies of 75 and $20 \mathrm{~Hz}$, respectively [15]. Then, the human body was discretized into rigid bodies and joints. Since the main motion during running occurs in lower extremities, legs were discretized well but the head-arms-trunk (HAT) model was used to model the upper body for the sake of simplicity. The RSP was also discretized into rigid links and rotational spring dampers [17]. Mass and inertial properties of each limb were estimated by using the anthropometrical measurements chart [18]. Position of joint locations were defined as simple relations between markers as given in Fig. 1 and local orientation axes were created by using markers on each segment.

Euler angles were calculated by using local coordinate systems between two rigid bodies. After calculation of joint angles, body and marker locations were reconstructed, because each limb lengths vary by time due to soft tissue movement, measurement errors, etc. The length of each limb is adjusted to remove kinematic inconsistency [19]. Mean values of the curves are assumed as lengths of each limb. MATLAB $\circledast$ (The Mathworks, Natick, USA) was used to process the data for reconstruction of the human body and motion, and to calculate joint angles.

The RSP was discretized into 6 rigid bodies connected by revolute joints and spring-damper elements between rigid bodies to include elasticity effect to structure. Mechanical properties of the RSP (E91 Runner, Ottobock, Duderstadt, Germany) were investigated by the force-displacement characteristics obtained by compressive testing. Force was applied at mounting position of the RSP and horizontal motion of the tip was limited by using a fixture. Then, stiffness of each spring between rigid segments was adjusted by considering design parameters of the RSP, stiffness in vertical direction, height, width and toe length as shown in the Fig. 2. 9 running blade was designed to investigate the effect of parameters on human behavior. Stiffness and damping parameters of the prosthesis were updated for each blade.

\section{Forward and Inverse Dynamic Analyses}

A computational model of the human body was created by using joint definitions, joint angles and inertial properties obtained in previous steps. First, we carried out forward dynamic analyses to determine kinematical accuracy. Force and kinematic data were imposed to model in order to run forward dynamic analyses. Modeling and analyses were carried out by using Altair MotionView and Altair MotionSolve. Kinematic measures, such as joint angle and length of limbs, and stiffness and damping coefficient of the RSP was validated by forward dynamic analyses. Since the damping of the prosthesis is dynamic characteristics of the structure, damping coefficients of the spring-damper elements were also adjusted in this step. Global positions of right and left knee were used to evaluate the model to validate the model. According to Fig. 3, the model exhibits similar behavior with experimental results. Asymmetry of motion can be observed by considering positions of prosthetic and sound leg knee positions. Larger 
displacement occurred in the position of prosthetic leg knee joint compared to sound legs. The difference between experimental and computational model occurs due to soft tissue movements, kinematic constraints, measurement errors and fixed center of pressure point on RSP and foot in forward dynamics calculations.

Inverse dynamic analyses were carried out to predict the sprint performance of the runner with different RSP. GRFs were estimated by using a contact model. Impact contact model was used to define the interaction between foot-ground and prosthesis-ground. Spheres were used to define contacting geometries of foot and prosthesis. The model was validated by comparing experimental and computational GRFs, also knee positions as it was done in forward dynamic analysis. According to results given in Figs. 4 and 5, contact time, maximum peak force in vertical and horizontal directions for prosthetic and sound leg, also curve trends of GRFs express similar characteristics. Besides, positions of knee joints have similar trends with experimental results. Error in inverse dynamics results is lower than error in forward dynamics results in some regions because center of pressure can move due to contact model and accuracy of modelling increases.

\section{Response Surface Method for Motion and Performance}

Understanding the effect of each shape/stiffness parameter of RSP on design and motion facilitate to increase the performance of runners. Response surface method was used to learn how the motion of joints and performance of runner change depending on the RSP. Analyses of the various prostheses in the design domain were conducted and various results were acquired.

The motion of human body adapts itself regarding the prosthesis used. It is important to understand how motion is going to change. Motion data of all trials ( 9 blades, 4 trials for each blade) were collected and joint angles were calculated. The motion of joints was assumed as periodic motion and period was calculated as one gait cycle. Then, the motion of joints was normalized by time to evaluate characteristics. Second-order polynomial response surfaces were created to approximate the effect of each parameter on period and joint motion in the sagittal plane. Finally, the approximated motion of humans considering the parameters of prosthesis used was obtained. The effect of each parameter on prosthetic knee joint angle is given in Fig. 6 .

After creating response surfaces of motion, new computational models were created. When shape and stiffness of prostheses changes, stiffness and damping ratio of joints in the multi-link prosthesis model is updated to run analyses correctly. Finally, computational models in the design domain were created and inverse dynamic analyses of all models were repeated to obtain responses. 4 different objective functions were created to evaluate the sprint performance of running trials. Since one of the main aims of the study is to develop sprint performance of the runner, performance-related design functions were defined. These functions were horizontal velocity change over one period, average horizontal velocity, horizontal and vertical impulse of ground reaction forces. After obtaining outputs of horizontal position and velocity of center of mass of the runner, and GRFs for all models in design space, response surfaces were created to hold information about the effect of each RSP design parameter. 


\section{Results And Discussions}

Response surface of horizontal velocity change over one period is given in figure 7. An increase in stiffness and width in the design domain leads the runner to have better sprint performance. Height and toe length have less effect on the sprint performance in unilateral amputees. It is concluded that bending stiffness distribution along the blade, contacting area and joint position of the multi-link model of the RSP were adjusted through determining the RSP parameters to improve sprinting performance.

Horizontal velocity change over one period and average horizontal velocity shows similar responses; however, the behavior of GRF impulse-related functions was slightly different. Therefore, an evaluation parameter was proposed to investigate the effect of both vertical and horizontal impulses as single value. Bouncing ball analogy was used to derive an evaluation parameter by simplifying the motion of the runner as given in figure 8 . It was assumed that longer distance after each contact will increase performance. The human body was considered as mass, initial velocities and, in the horizontal and vertical axis, respectively. In the vertical direction, velocities before and

Response surface of horizontal velocity change over one period is given in figure 7 . An increase in stiffness and width in the design domain leads the runner to have better sprint performance. Height and toe length have less effect on the sprint performance in unilateral amputees. It is concluded that bending stiffness distribution along the blade, contacting area and joint position of the multi-link model of the RSP were adjusted through determining the RSP parameters to improve sprinting performance.

Horizontal velocity change over one period and average horizontal velocity shows similar responses; however, the behavior of GRF impulse-related functions was slightly different. Therefore, an evaluation parameter was proposed to investigate the effect of both vertical and horizontal impulses as single value. Bouncing ball analogy was used to derive an evaluation parameter by simplifying the motion of the runner as given in figure 8 . It was assumed that longer distance after each contact will increase performance. The human body was considered as mass, initial velocities and, in the horizontal and vertical axis, respectively. In the vertical direction, velocities before and after contact were assumed as same magnitude, but in opposite direction. Aerial time was obtained by calculating velocity in the vertical direction. Then, the change of velocity in the horizontal direction was calculated by using the horizontal impulse. If initial velocities were assumed the same, the final velocity will be directly related to the change of velocity. Then, evaluation parameter was obtained by omitting initial horizontal velocity term from horizontal distance. It was revealed that not only vertical impulse, but also horizontal impulse should be considered together to understand performance. 


$$
\begin{gathered}
t_{\text {aerial }}=\frac{2 u}{g}=\frac{I_{\text {ver }}}{m g} \\
v_{\text {new }}=\frac{I_{\text {hor }}}{m}-v_{\text {old }} \\
\text { Horizontal distance }=\frac{I_{\text {ver }}}{m g} \times\left(\frac{I_{\text {hor }}}{m}-v_{\text {old }}\right) \\
\text { Evaluation parameter }=\frac{I_{\text {ver }} I_{\text {hor }}}{m^{2} g}
\end{gathered}
$$

It was concluded that the change of shape and stiffness of the RSP affects the performance of athletes. Therefore, the choice of prosthesis holds an important place for individuals to perform well.

On the other hand, knee-buckling can be a risk for the transfemoral amputees who use artificial knee joints. Therefore, the knee buckling risk should be considered in the RSP design and optimization process. Knee-buckling occurs when moment exists in the direction of knee flexion at stance phase. The relative position of the artificial knee with respect to the center of pressure and ground reaction force vectors decides the direction of the joint moment around knee joint. Toe length is the parameter that changes bottom half of the shape of the prosthesis, thus, the effect of RSP toe length on prosthetic knee moment was investigated. As it can be seen in figure 9 , the reduction of toe length causes a smaller moment in the opposite direction of knee flexion. In other words, it is easier to observe knee buckling if RSP toe length is reduced. On the other hand, an increase in toe length may cause a different type of trouble, such as tripping during swing phase. Since it may be a risk for fall, therefore the closest position to the ground was obtained. According to figure 10, when toe length increases, trajectory of the RSP become closer to ground. Therefore, an increase in toe length also increases the risk of touch down to the ground while swinging.

after contact were assumed as same magnitude, but in opposite direction. Aerial time was obtained by calculating velocity in the vertical direction. Then, the change of velocity in the horizontal direction was calculated by using the horizontal impulse. If initial velocities were assumed the same, the final velocity will be directly related to the change of velocity. Then, evaluation parameter was obtained by omitting initial horizontal velocity term from horizontal distance. It was revealed that not only vertical impulse, but also horizontal impulse should be considered together to understand performance.

It was concluded that the change of shape and stiffness of the RSP affects the performance of athletes. Therefore, the choice of prosthesis holds an important place for individuals to perform well.

On the other hand, knee-buckling can be a risk for the transfemoral amputees who use artificial knee joints. Therefore, the knee buckling risk should be considered in the RSP design and optimization 
process. Knee-buckling occurs when moment exists in the direction of knee flexion at stance phase. The relative position of the artificial knee with respect to the center of pressure and ground reaction force vectors decides the direction of the joint moment around knee joint. Toe length is the parameter that changes bottom half of the shape of the prosthesis, thus, the effect of RSP toe length on prosthetic knee moment was investigated. As it can be seen in figure 9 , the reduction of toe length causes a smaller moment in the opposite direction of knee flexion. In other words, it is easier to observe knee buckling if RSP toe length is reduced. On the other hand, an increase in toe length may cause a different type of trouble, such as tripping during swing phase. Since it may be a risk for fall, therefore the closest position to the ground was obtained. According to figure 10, when toe length increases, trajectory of the RSP become closer to ground. Therefore, an increase in toe length also increases the risk of touch down to the ground while swinging.

\section{Conclusion}

In this study, a methodology to design the RSP was proposed to improve sprinting performance of runners. It was revealed that the RSP design affects running kinematics, sprinting performance, possibility of knee buckling and tripping. A 3-dimensional model of a unilateral transfemoral amputee was created. Forward and inverse dynamic analyses of runner were carried out and validated by using the joint positions and ground reaction forces. Then, joint angles for all trials were calculated by using 3-D motion capture data. Changes of human behavior due to different design parameters of the prosthesis were approximated by using response surface method and new computational models were generated by using estimated motion data. The sprint performance was evaluated by acceleration related functions. Knee buckling and tripping during the swing phase of the prosthetic leg were considered as limitations. Finally, the case-optimal prosthesis regarding 4 different performance functions was obtained and it was revealed that higher stiffness and width give better performance for the runner. While high toe length increases the risk of the tripping in prosthetic leg, low toe length increases the risk of knee buckling. To conclude, the prosthesis has a significant effect on the sprint performance of athlete with RSP and choice of the prosthesis for a runner is important. Especially, unilateral amputee runners, because of running asymmetry, develop different strategies to run faster and keep balance depending on prosthesis they used. Response surface method can be used to understand how shape and stiffness of prosthesis affect the motion of joints they adapt.

\section{Abbreviations}

RSP

Running-specific prosthesis

GRF

Ground reaction force

FEM

Finite element method 
HAT

Head-Arms-Trunk

\section{Declarations}

\section{Availability of data and materials}

The datasets used and/or analyzed during the current study are available from corresponding author on reasonable request.

\section{Competing Interests}

The authors declare that they have no competing interest.

\section{Funding}

Not applicable

\section{Authors' Contributions}

In this study, CG contributed to the development of model and method, calculations, and investigation of the performance. $\mathrm{HH}$ contributed to experiments and helped biomechanical discussions. $\mathrm{KS}, \mathrm{HH}, \mathrm{SS}$ helped to discussions and evaluation of results. All authors read and approved the final manuscript.

\section{Acknowledgements}

Not applicable

\section{References}

1. Nolan $L$ (2008) Carbon fibre prostheses and running in amputees: A review. Foot Ankle Surg 14(3):125-129. doi:https://doi.org/10.1016/j.fas.2008.05.007

2. Hobara H, Baum BS, Kwon HJ, Miller RH, Ogata T, Kim YH, Shim YK (2013) Amputee locomotion: Spring-like leg behavior and stiffness regulation using running-specific prostheses. J Biomech 46(14):2483-2489. doi:https://doi.org/10.1016/j.jbiomech.2013.07.009

3. Fey NP, Klute GK, Neptune RR (2013) Altering prosthetic foot stiffness influences foot and muscle function during below-knee amputee walking: A modeling and simulation analysis. J Biomech 46(4):637-644. doi:https://doi.org/10.1016/j.jbiomech.2012.11.051 
4. Rigney SM, Simmons A, Kark L (2015) Concurrent multibody and Finite Element analysis of the lower-limb during amputee running. In: 2015 37th Annual International Conference of the IEEE Engineering in Medicine and Biology Society (EMBC)

5. Beck ON, Taboga P, Grabowski AM (2017) How do prosthetic stiffness, height and running speed affect the biomechanics of athletes with bilateral transtibial amputations? J R Soc Interface 14(131):20170230. doi:10.1098/rsif.2017.0230

6. Sano Y, Makimoto A, Hashizume S, Murai A, Kobayashi Y, Takemura H, Hobara H (2017) Leg stiffness during sprinting in transfemoral amputees with running-specific prosthesis. Gait Posture 56:65-67. doi:https://doi.org/10.1016/j.gaitpost.2017.04.038

7. Rigney SM, Simmons A, Kark L (2017) Mechanical characterization and comparison of energy storage and return prostheses. Med Eng Phys 41:90-96.

doi:https://doi.org/10.1016/j.medengphy.2017.01.003

8. Grabowski AM, McGowan CP, McDermott WJ, Beale MT, Kram R, Herr HM (2010) Running-specific prostheses limit ground-force during sprinting. Biol Lett 6(2):201-204. doi:10.1098/rsbl.2009.0729

9. Sepp LA, Baum BS, Nelson-Wong E, Silverman AK (2019) Dynamic balance during running using running-specific prostheses. J Biomech 84:36-45.

doi:https://doi.org/10.1016/j.jbiomech.2018.12.016

10. Baum BS, Borjian R, Kim YS, Linberg A, Shim JK (2010) Optimization and Validation of a Biomechanical Model for Analyzing Running-Specific Prostheses. In: Herold KE, Vossoughi J, Bentley WE (ed) 26th Southern Biomedical Engineering Conference SBEC 2010, April 30 - May 2, 2010, College Park, Maryland, USA, IFMBE Proceedings, vol 32. Springer, Berlin, Heidelberg

11. Murai A, Hobara H, Hashizume S, Kobayashi Y, Tada M (2018) Can forward dynamics simulation with simple model estimate complex phenomena?: Case study on sprinting using running-specific prosthesis. Robomech J 5(1):10. doi:10.1186/s40648-018-0108-8

12. Jackson JN, Hass CJ, Fregly BJ (2016) Development of a Subject-Specific Foot-Ground Contact Model for Walking. J Biomech Eng 138(9). doi:10.1115/1.4034060

13. Fey NP, Klute GK, Neptune RR (2012) Optimization of Prosthetic Foot Stiffness to Reduce Metabolic Cost and Intact Knee Loading During Below-Knee Amputee Walking: A Theoretical Study. J Biomech Eng 34(11):111005-111010. http://dx.doi.org/10.1115/1.4007824

14. Yang L, Dyer PS, Carson RJ, Webster JB, Bo Foreman K, Bamberg SJM (2012) Utilization of a lower extremity ambulatory feedback system to reduce gait asymmetry in transtibial amputation gait. Gait Posture 36(3):631-634. doi:https://doi.org/10.1016/j.gaitpost.2012.04.004

15. Namiki Y, Hashizume S, Murai A, Kobayashi Y, Takemura H, Hobara H (2019) Joint moments during sprinting in unilateral transfemoral amputees wearing running-specific prostheses. Biol Open 8(2):bio039206. doi:10.1242/bio.039206

16. van Sint Jan S (2007) Color Atlas of Skeletal Landmark Definitions. Churchill Livingstone, London

17. Rigney SM, Simmons A, Kark L (2016) A prosthesis-specific multi-link segment model of lower-limb amputee sprinting. J Biomech 49(14):3185-3193. 
doi:https://doi.org/10.1016/j.jbiomech.2016.07.039

18. Winter DA (2009) Anthropometry. In: Biomechanics and Motor Control of Human Movement. John Wiley \& Sons, Inc, Hoboken, pp 82-106

19. Pàmies Vilà $R$ (2012) Application of multibody dynamics techniques to the analysis of human gait. TDX (Tesis Dr en Xarxa). http://www.tdx.cat/handle/10803/123774. Accessed February 19, 2019

\section{Figures}

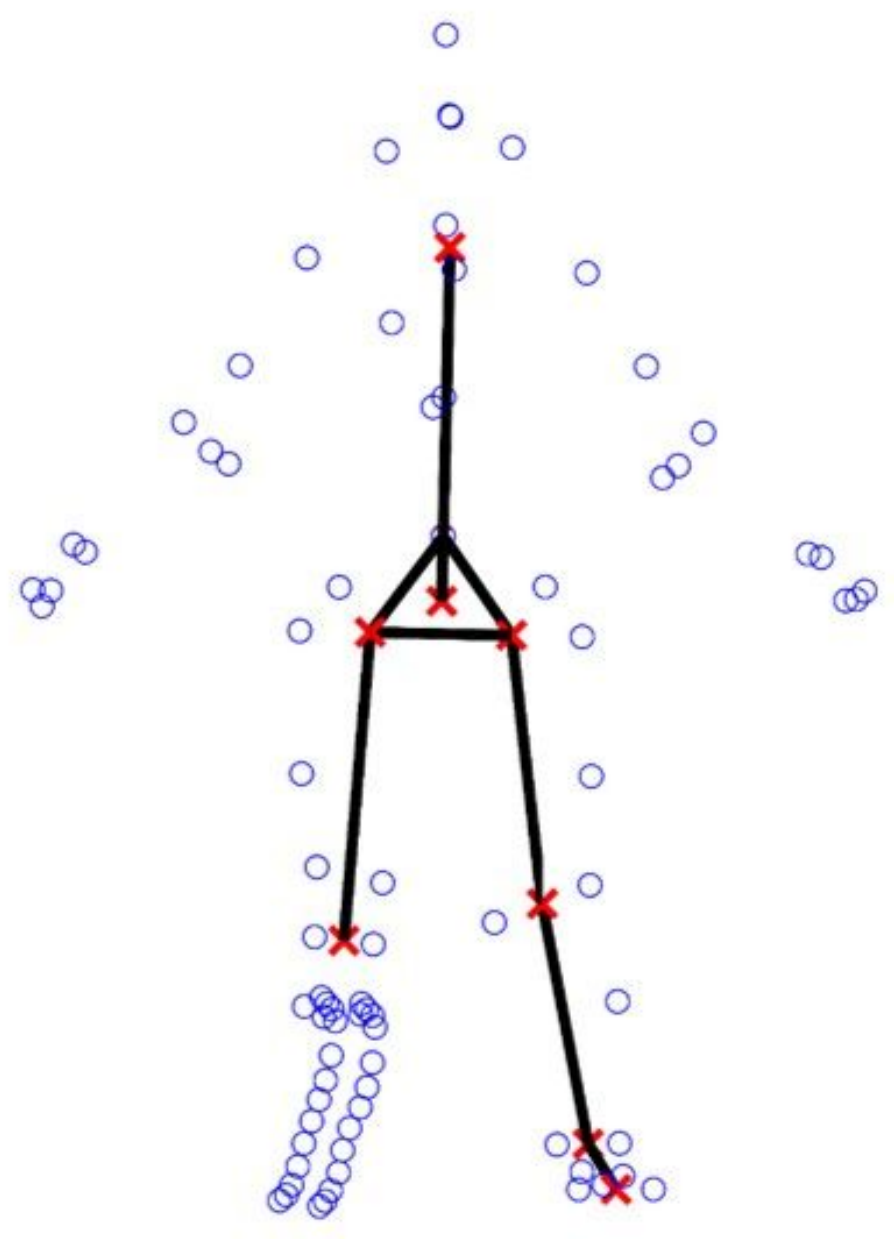

Figure 1

Marker positions and rigid links on the human body. Blue circles, red crosses and black lines represent marker locations, joint locations and body segments, respectively. 


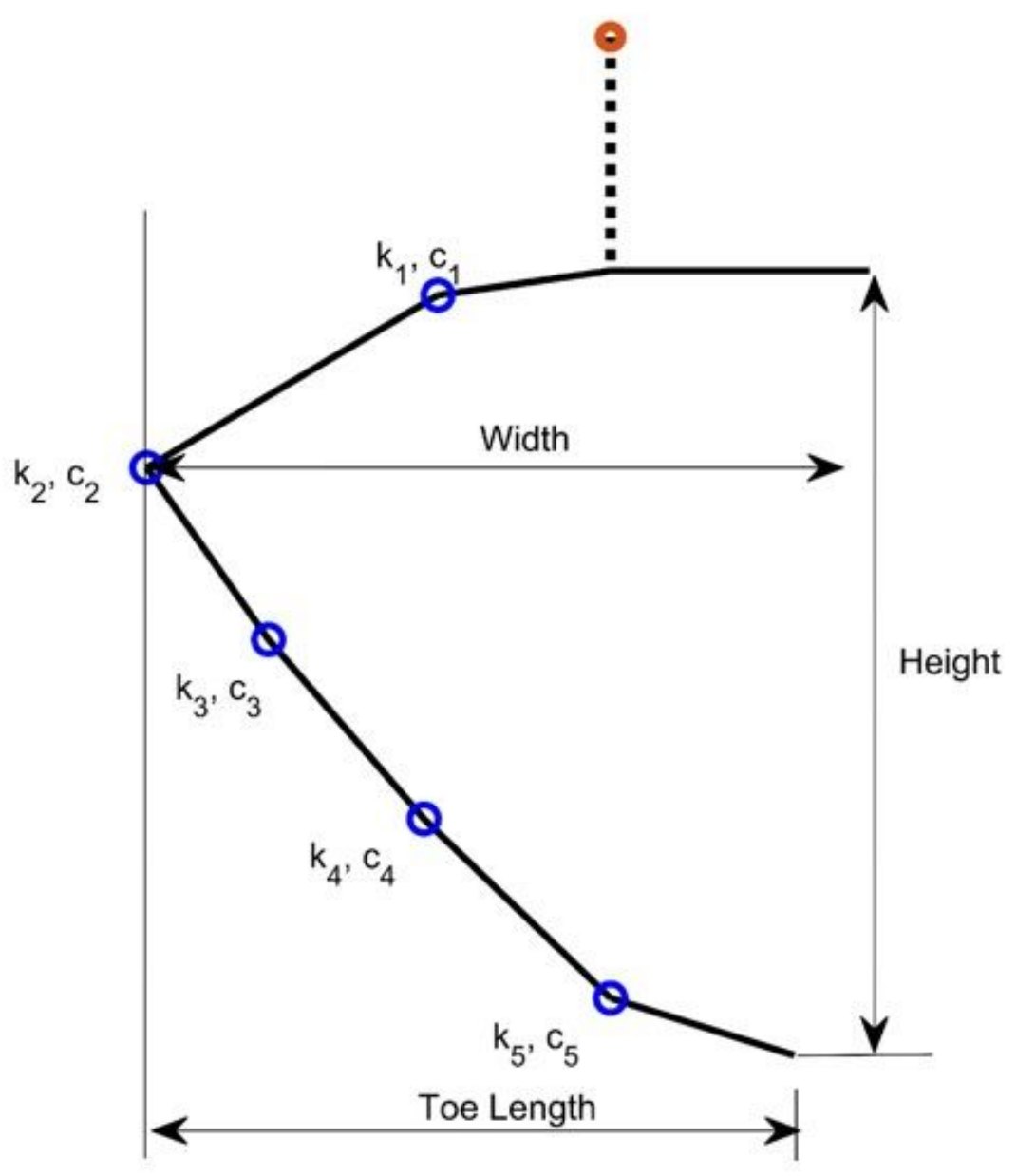

Figure 2

Definition of the RSP design parameters. The red circle and blue circles represent prosthetic knee joint and joints of the RSP multilink model, respectively. Solid black lines symbolize rigid links of the multilink model and dashed black line represents connection between carbon fiber prosthesis and prosthetic knee joint. 


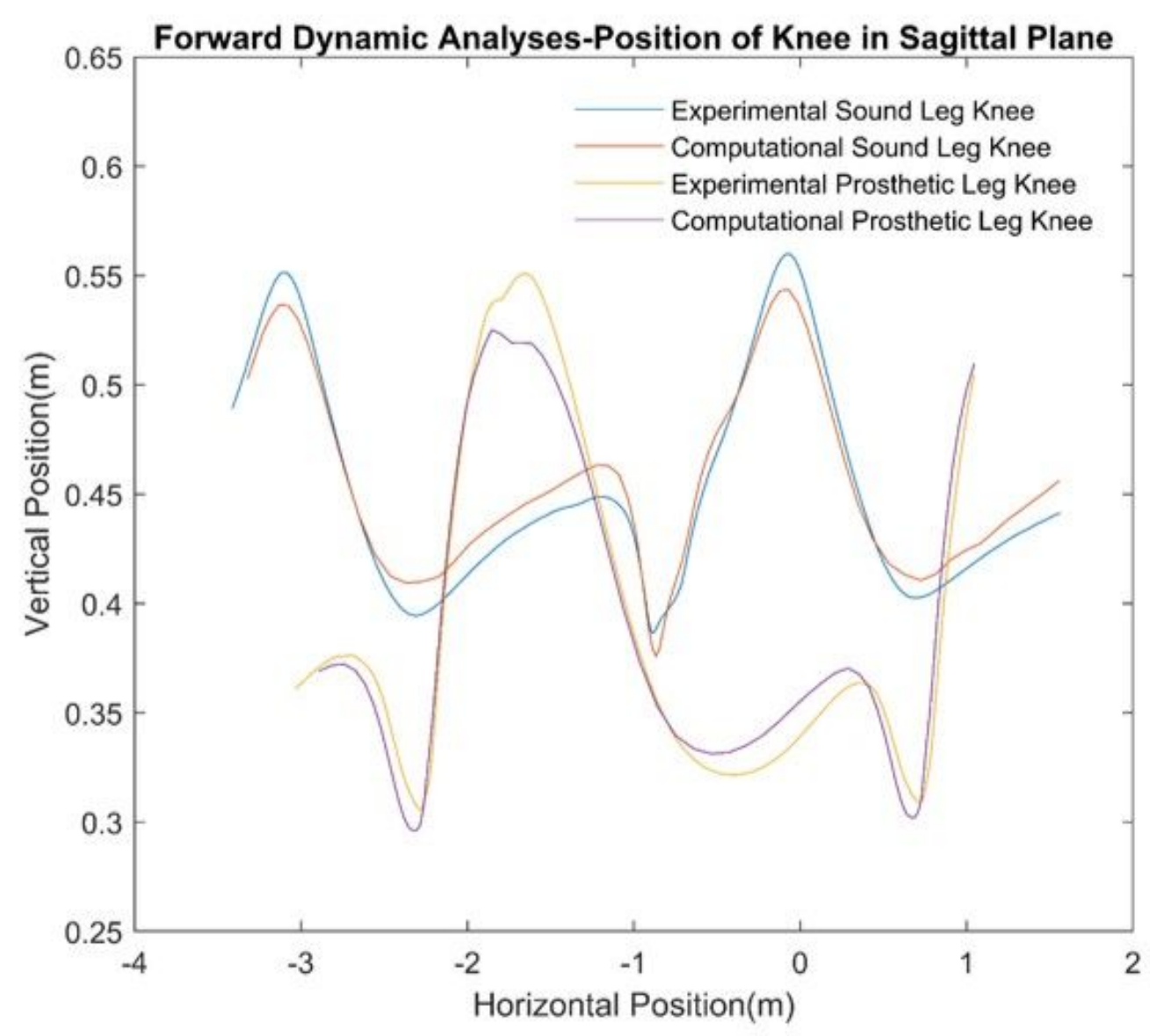

Figure 3

Comparison of knee joint positions obtained by experimental and forward dynamic analyses results. 


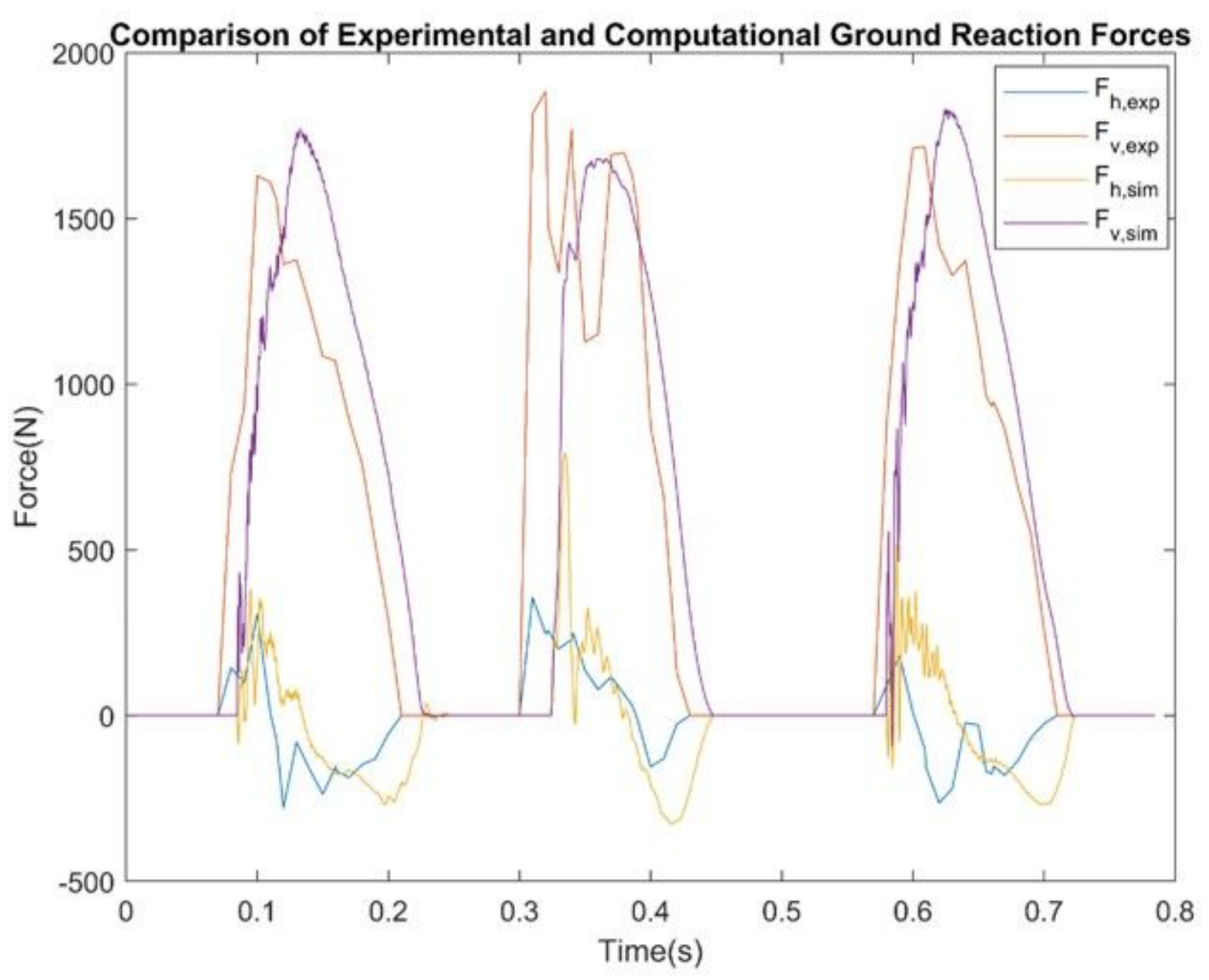

Figure 4

Comparison of experimental and computational ground reaction forces of the RSP contact, sound foot contact and RSP contact 


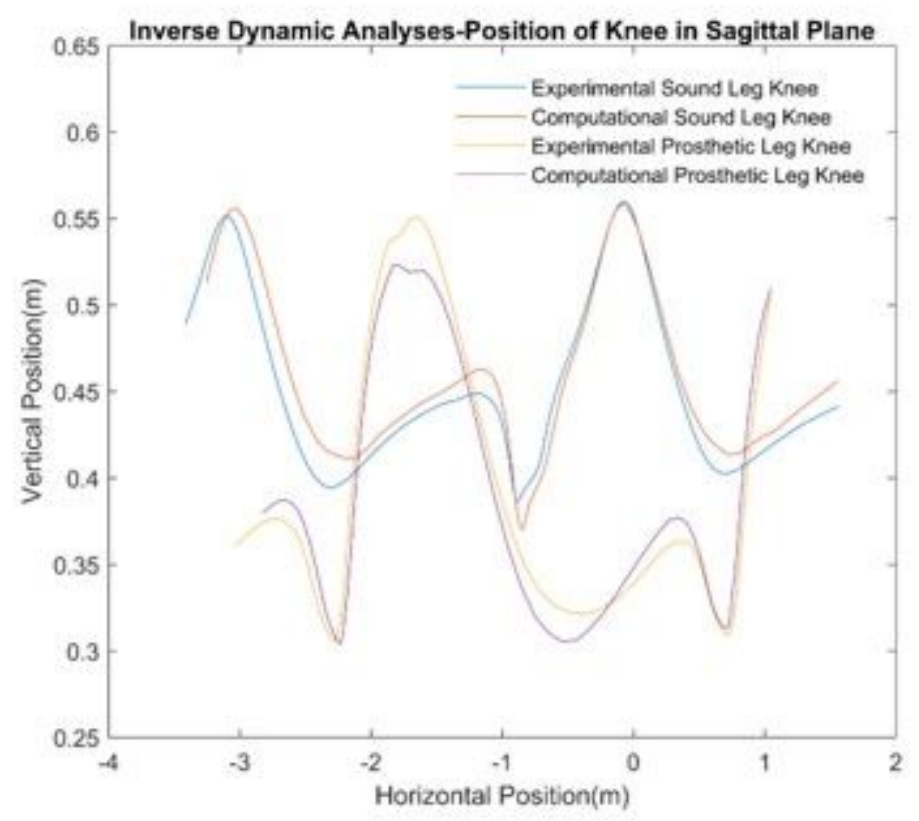

Figure 5

Comparison of knee joint position obtained by experiment and inverse dynamic analyses
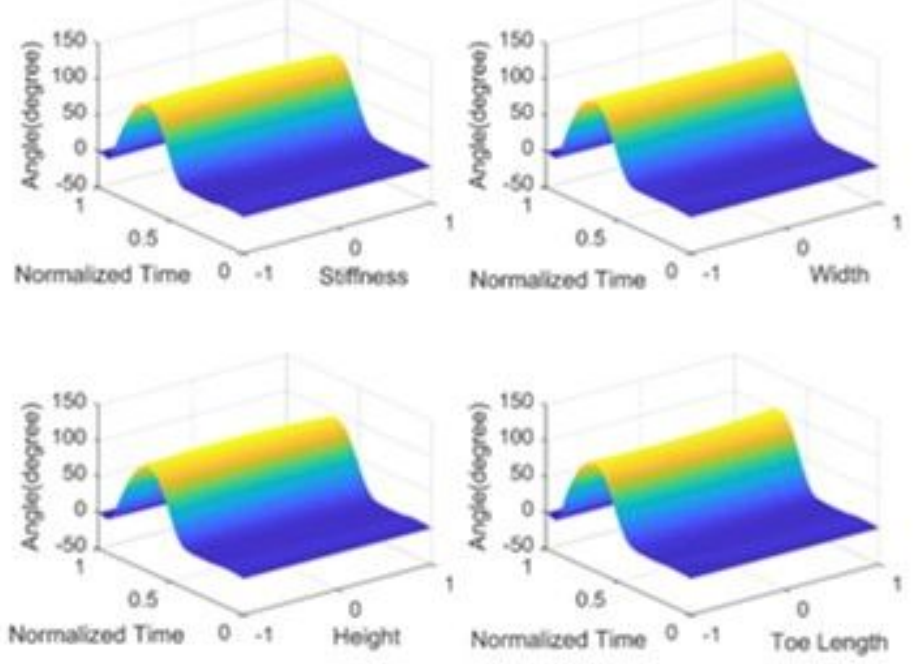

\section{Figure 6}

Variations of prosthetic knee joint angle in sagittal plane with respect to RSP design parameters: stiffness, width, height and toe length in normalized time. Interval of $[-1,1]$ represents minimum and maximum values in the design domain of each design parameter. 

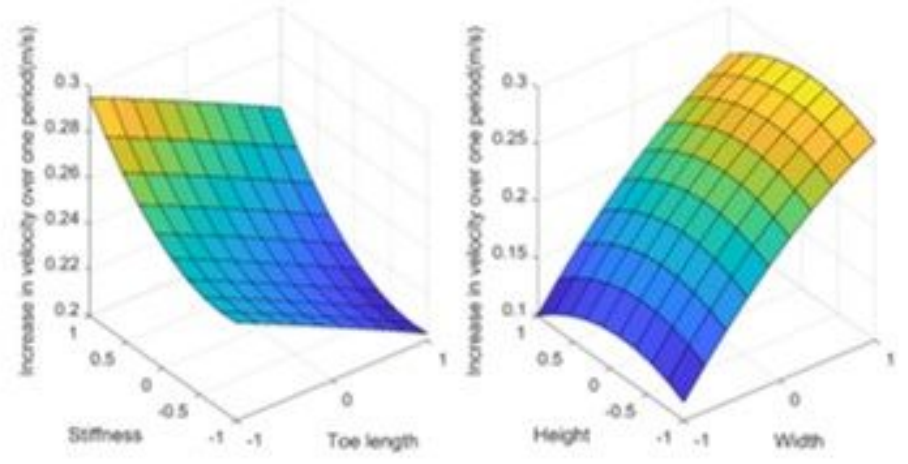

\section{Figure 7}

Change of the horizontal velocity over one period with respect to RSP design parameters. Interval of $[-1,1]$ represents minimum and maximum values in the design domain of each parameter.

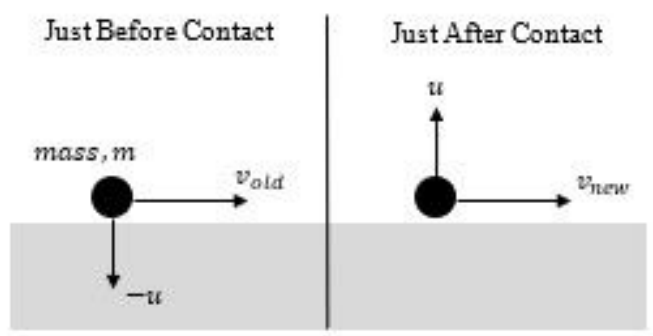

\section{Figure 8}

Bouncing ball analogy is considered to evaluate the performance of different prostheses. Velocities just before and after contact is calculated by using the relation between impulse and momentum. 


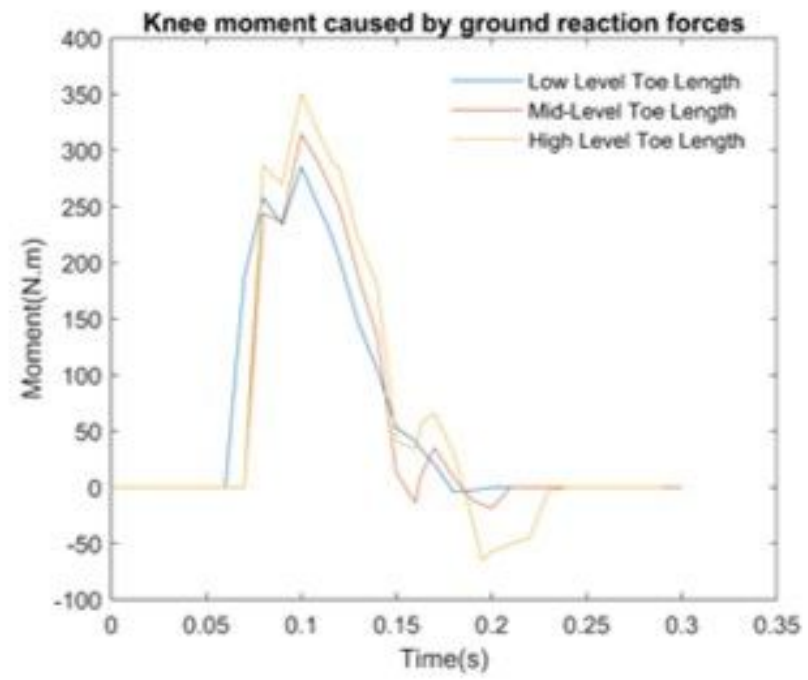

Figure 9

Effect of RSP toe length on knee moment.

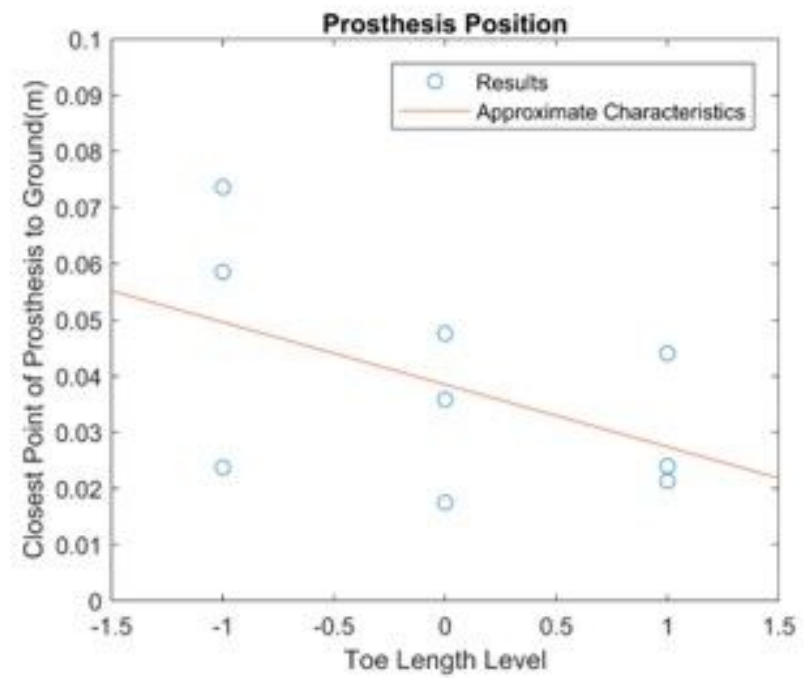

Figure 10

Effect of RSP toe length on the closest distance to ground while swinging 